\title{
Investigation on the Dynamic Behavior of a Solenoid Hydraulic Valve for Automotive Semi-Active Suspensions Coupling 3D and 1D Modeling
}

\author{
Matteo Pelosi, Kashyap Subramanya*, and Jonas Lantz** \\ Öhlins Racing AB, Jönköping, Sweden \\ *Linköping University, Linköping, Sweden \\ **Applied Thermodynamics and Fluid Mechanics Division, Linköping University, Linköping, Sweden \\ E-mail: matteo.pelosi@ohlins.se,kassu303@student.liu.se,jonas.lantz@liu.se
}

\begin{abstract}
The aim of this paper is to investigate the influence of the internal fluid flow characteristics of a special hydraulic solenoid valve, developed by Öhlins Racing AB, on its overall dynamic behavior. This valve is a two stage hydraulic pressure control valve and is typically mounted on each shock absorber of an on-road vehicle, allowing the implementation of semi-active suspension functionality. This technology is referred as CES (Continuously Controlled Electronic Suspension). The CES valve allows continuously controlling the vehicle shock absorbers damping characteristic by proportionally adjusting the metering geometry offered to its damping element, i.e. hydraulic oil. The electronic valve actuation and control, obtained through an electromagnetic solenoid, is based on the input from several vehicle dynamics sensors, such as accelerometers, gyroscopes and other displacement sensors. The CES valve's unconventional design significantly influences the fluid flow, making the use of numerical modeling essential to discover its physical behavior and to support further product development. In this paper, a CFD (Computational Fluid Dynamics) analysis on the main and pilot stages of the hydraulic valve is discussed. This 3D numerical analysis is used to extract critical physical variables, affecting the valve behavior, such as flow coefficients and pressure distributions on the moving elements, i.e. flow forces. This information is coupled with a detailed lumped parameter model of the hydraulic valve, which solves for the valve moving element dynamics considering the action of the main external forces. Moreover, the 1D model allows predicting the valve critical pressure/flow characteristics. It is shown how the coupling of 3D modeling results with the CES valve 1D model strongly improves the whole valve dynamics numerical predictions over traditional methods for considering the effect of fluid inertia and discharge in lumped parameter simulations. Comparisons with measurement both on single regions of the CES hydraulic valve and on the entire valve are discussed in order to validate the various phases of numerical modeling.
\end{abstract}

Keywords: Semi-active suspensions, shock absorber, hydraulic valve, flow forces, CFD

\section{Introduction}

Several aspects influencing the behavior of two-wheel or four-wheel vehicles, both on-road and off-road, are significantly influenced by their shock absorbers characteristics. In particular, the vehicle comfort, handling, grip and stability are a direct consequence of the shock absorber performance and they have to be carefully tuned by a vehicle manufacturer in order to meet the customer demands.

Most of the vehicles today are equipped with passive shock absorbers, which, by connecting the vehicle chassis to the wheels, perform a critical damping function on the overall vehicle dynamics, leading to an improved ride quality. Referring to conventional shock absorbers, the damping characteristic is typically obtained by metering hydraulic oil through a set of special internal valves mounted in the damper. The damper kinetic energy is dissipated through fluid friction and the shock absorber damping response depends on the passive valves tuning. Different damping behavior for the shock absorbers compression (wheel moving towards the chassis) and rebound (wheel moving away from the chassis) cycles can be implemented, by mounting valves with different metering characteristics.

Öhlins Racing AB (Sweden) is a worldwide leader in the design and development of passive shock absorbers for a variety of on-road and off-road applications. These state of the art shock absorbers fulfill the vehicle damping requirements, by allowing the end customer a wide range of discrete settings while preserving a superior level of 
performance. However, a passive damper is unable to change its damping characteristics in real-time. The behavior of the shock absorber is determined by the hydraulic valving fixed metering settings and cannot continuously adapt to the changing driving conditions.

A hydraulic semi-active suspension overcomes this limitation, by allowing a continuous electronic control of the shock absorber damping characteristics. This is obtained by continuously varying the metering geometry offered to hydraulic oil within the damper. The semi-active shock absorber can adapt its behavior during the compression and rebound cycles, considering several driving aspects, i.e. vehicle roll, pitch and yaw attitudes, vehicle accelerations, wheel displacements and throttle position. A simplified depiction of a semi-active suspension system is shown by Figure 1.

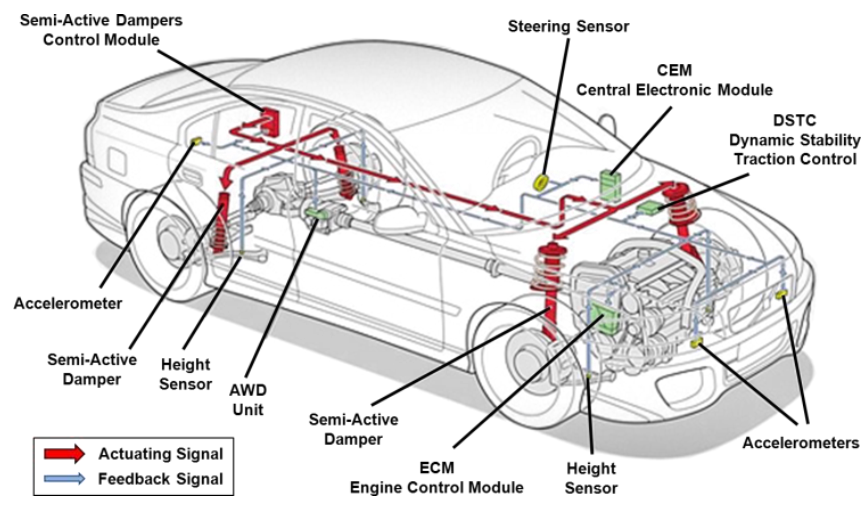

Figure 1: Semi-active suspension technology schematic

Öhlins CES Technologies is dedicated to the research and development of semi-active suspensions technology, named CES (Continuously Controlled Electronic Suspension). In particular, the core of Öhlins semi-active suspensions is represented by the hydraulic control valve responsible for continuously controlling the shock absorber fluid flow and damping performance. This hydraulic valve, is a two stage proportional control valve, actuated by an electromagnetic solenoid. It represents a state of the art component, in use by the several major automotive manufacturers to offer semiactive suspensions as standard or optional features in their vehicles. The special hydraulic valve design allows obtaining a smooth pressure-flow characteristic at different input currents, which is ideal for implementing efficient damper control strategies. To obtain such performance characteristics, the CES valve presents unique design features, which strongly influences the internal fluid flow behavior. In addition, the small dimensions of this component, coupled with high operating pressure and flow rates, make several design details critical for a proper valve performance. Therefore, only through advanced computer simulation it is possible to capture the physical phenomena taking place in the valve, to exactly predict its behavior, to study novel design changes and to shorten the product development leading to a better performance by supporting Öhlins Racing engineers during the design phase.

For these reasons, in this paper a CFD (Computational Fluid Dynamics) analysis on the most critical regions of the CES valve is presented. The numerical results obtained by this extensive 3D modeling analysis are discussed and used to improve the predictions of a detailed lumped parameter model of the CES valve. In fact, if the steady state CFD studies allow capturing the internal fluid behavior at different boundary conditions and moving elements positions, only with a $1 \mathrm{D}$ numerical model it is possible to predict the overall valve dynamic performance, both when studied singularly or coupled with a shock absorber model. In particular, the CFD results allow defining the correct flow regime and flow coefficients at different operating conditions for the critical metering elements of the valve. In addition, the prediction of the flow field static pressure distribution on the moving element surfaces is a key to determine their correct force balance [1]-[3]. Hence, the results from CFD analysis are used to consider the effect of flow inertia on the moving elements dynamic equilibrium solved by the lumped parameter model.

The numerical results obtained by the coupled 3D and 1D numerical modeling are validated both by using special measurements on specific regions of the valve and by testing the complete CES valve over its typical working range.

\section{The CES8700 Solenoid Hydraulic Valve}

In this study, one of the CES valves designed by Öhlins CES Technologies, named CES8700, is analyzed and is shown in Figure 2.
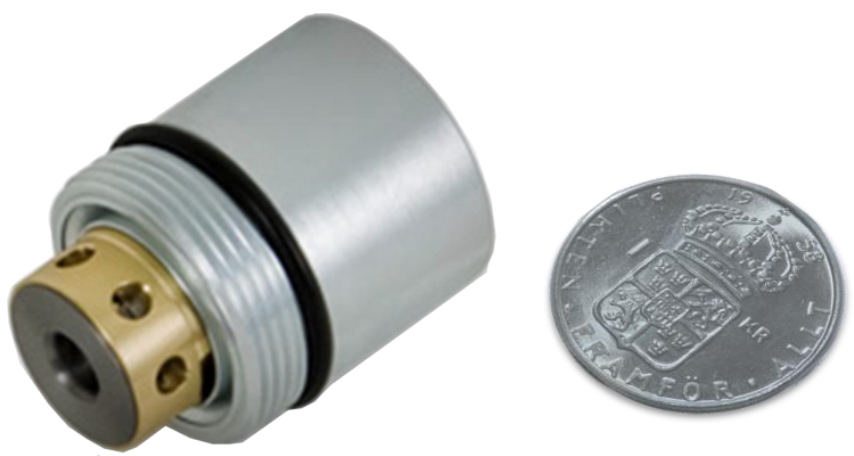

Figure 2: Öhlins Racing CES8700 hydraulic control valve compared to 1 Swedish Krona

The CES8700 is a pilot operated pressure control valve composed by two hydraulic stages, a main stage and a pilot stage, and continuously controlled by an electromagnetic linear actuator, a solenoid.

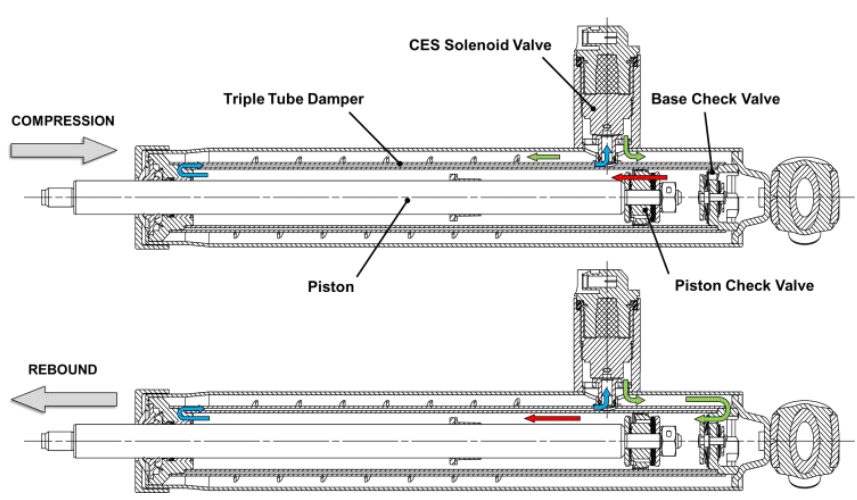

Figure 3: CES8700 valve operation in triple tube damper 
The CES8700 valve is externally mounted on triple tube type shock absorbers and, thanks to the triple tube design, receives flow only in one direction through the main stage inlet port, as depicted by Figure 3 .

The pilot operated solenoid valve allows controlling the damper fluid pressure through the main stage poppet using small solenoid forces. The pilot stage moving element, connected to the solenoid rod, by regulating the main stage pilot pressure, determines the main poppet metering effect on the damper fluid flow. Hence, a continuously controllable damper stiffness is achieved by adjusting the pressure forces acting on the piston of the shock absorber.

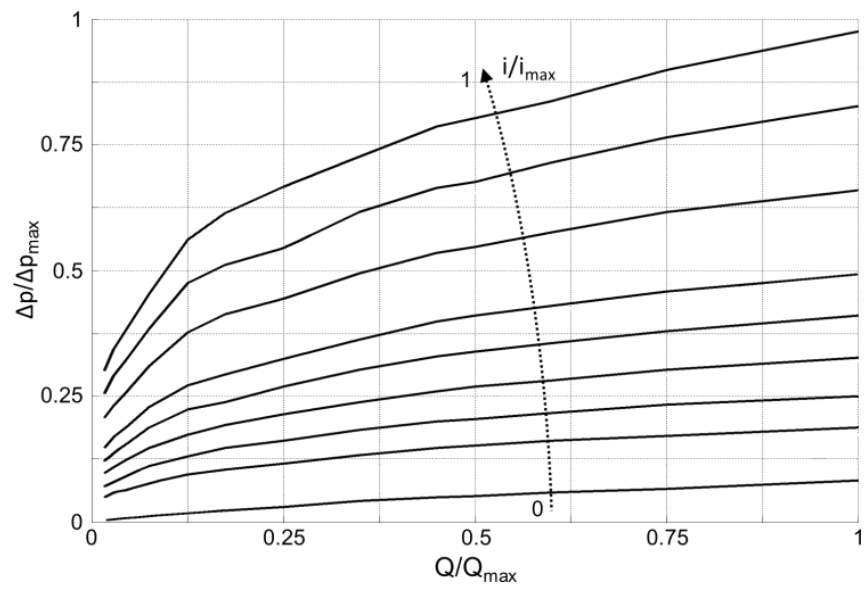

Figure 4: CES p-Q characteristic over a range of currents

A typical CES pressure/flow characteristic is shown in Figure 4. It can be noticed how increasing levels of input current generate a progressively higher pressure level across the valve. This is due to the higher solenoid force acting on the pilot poppet, determining higher pilot pressure and increasing the main poppet metering effect.

The understanding of the physical phenomena within the main stage and pilot stage is critical to predict the hydraulic valve behavior. Hence, the numerical modeling is focused on capturing the behavior of the critical features of these two stages. In particular, the key components of main and pilot stages are presented in Figure 5.

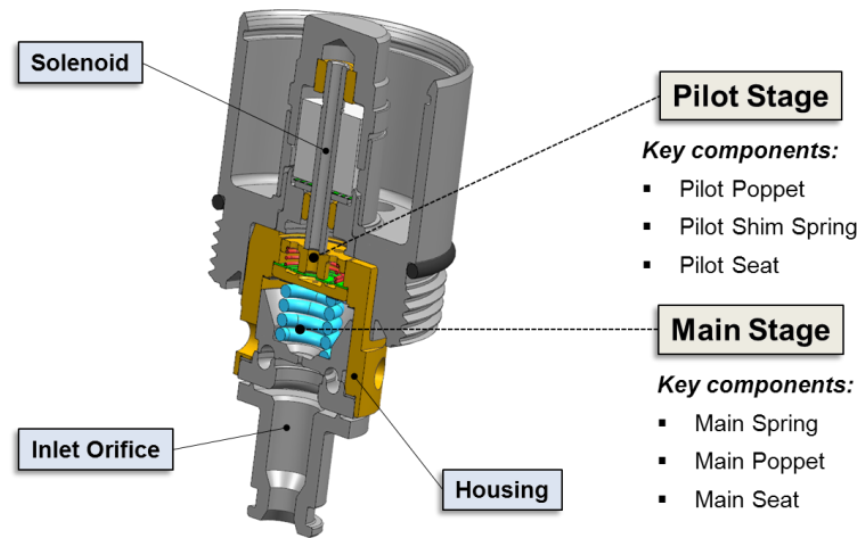

Figure 5:CES8700 detailed view of key mechanical components

The pilot stage metering element is also an essential elastic element, being a specially designed shim spring. The pilot shim spring, always in contact with the pilot poppet, alters its shape during the stroke due to the spring elastic deformation. The main poppet presents two annular orifices in series separated by a unique toroid shaped volume, which provides the CES valve with a smooth metering characteristic and additional stability.

Due to the unconventional geometrical design of the CES valve, numerical simulation is used to understand the hydraulic valve behavior over a wide range of operating points. A 1D AMESim model solves the second order dynamics of the hydraulic system, considering the mutual influence of several external forces on the moving elements and the hydraulic volumes transient pressure build-up. Springs, viscous, hydro-static and hydro-dynamic forces are calculated by the lumped parameter simulation model, as well as instantaneous flow rates and pressures. Steady-state distributed parameters analysis on the main and pilot stages of the valve, based on Ansys Fluent solver, is used to improve the prediction of this detailed 1D simulation model, concerning in particular flow discharge and hydrodynamic forces behavior [4]. In the next sections, the numerical analysis and the methods introduced to couple 3D and 1D simulations are discussed.

\section{The 3D Ansys Fluent Simulations}

In order to capture the fluid flow behavior in one of the key variable metering regions of the CES8700 valve, RANS (Reynolds Averaged Navier-Stokes) CFD analysis have been performed. The Ansys Fluent solver has been used to run a set of steady-state simulations for the main stage and pilot stage geometries [5]-[10]. The two geometries have been analyzed in two separate environments, assigning proper boundary conditions. Velocity inlets/pressure outlets boundaries are chosen in order to cover the known operating range. In addition, knowing the pilot and main poppet maximum strokes, several CFD studies have been completed at discrete values of progressively higher poppet positions. The main settings for the performed steady-state CFD analysis on the two valve stages are grouped in Table 1.

\begin{tabular}{|l|l|}
\hline Fluid model & Newtonian, incompressible \\
\hline Turbulence model & RANS, realizable k- $\varepsilon$ \\
\hline Near wall model & Enhanced Wall Treatment \\
\hline Schemes Accuracy & Second order \\
\hline Mesh type & Hybrid (Teth + Prism + Hex) \\
\hline Mesh size & $\sim 2$ million cells \\
\hline
\end{tabular}

Table 1: CFD analysis general settings

The fluid is hydraulic oil, considered Newtonian in its shear properties and incompressible in the considered operating range. The turbulence is captured by using a typical two equations model, the realizable $k$ - $\varepsilon$ type [11]. This model is used in many practical engineering flow calculations, thanks to its robustness, economy and accuracy for a wide range of turbulent flows. Additionally, the realizable version, compared to standard $k-\varepsilon$ models, improves the mathematical formulations around the definition of the critical Reynolds stresses, leading to a better prediction over 
flows characterized by strong streamline curvature, vortices and rotations. The CES8700 valve geometry induces these types of flow features. Near-wall turbulence modeling is another critical aspect of RANS CFD simulations, strongly dependent on the grid resolution. The Enhanced Wall Treatment (EWT) available in Ansys Fluent, makes the turbulence model relatively insensitive to the first cell wall distance parameter, $y^{+}$, combining a two-layer model with wall functions. Where the mesh is fine enough, $y^{+} \approx 1$, a traditional two-layer zonal model is used to predict boundary layer behavior; while where the near-wall mesh is coarser an enhanced wall function formulation is implemented [12]. This method allows overcoming the computational requirements imposed by a standard nearwall resolving turbulence model, which imposes a mesh sufficiently fine everywhere. In order to further reduce the computational expense, hybrid meshes have been generated, combining different meshing methods. In particular, hex dominant meshes have been used wherever possible, to strongly reduce the element count and enhance numerical schemes discretization. Only the most geometrically complex zones have been meshed using tetrahedrons. The near-wall fluid shear layers have been discretized using 12 prism cells normal to the layers.

In the next sections, additional details on the performed CFD analysis for the CES8700 main and pilot stages are provided together with the most significant numerical results.

\subsection{Main Stage}

The main stage fluid domain accurately replicates the 3D inlet geometry and, due to some internal non-symmetrical features, an axisymmetric analysis could not be performed. Hence, the fluid domain is studied in a half symmetric condition, in order to reduce the computational burden. Figure 6 provides a depiction of the main stage geometry and fluid domain. The hole connecting the inlet volume with the main poppet chamber is removed to isolate the analysis to the main stage restrictions.

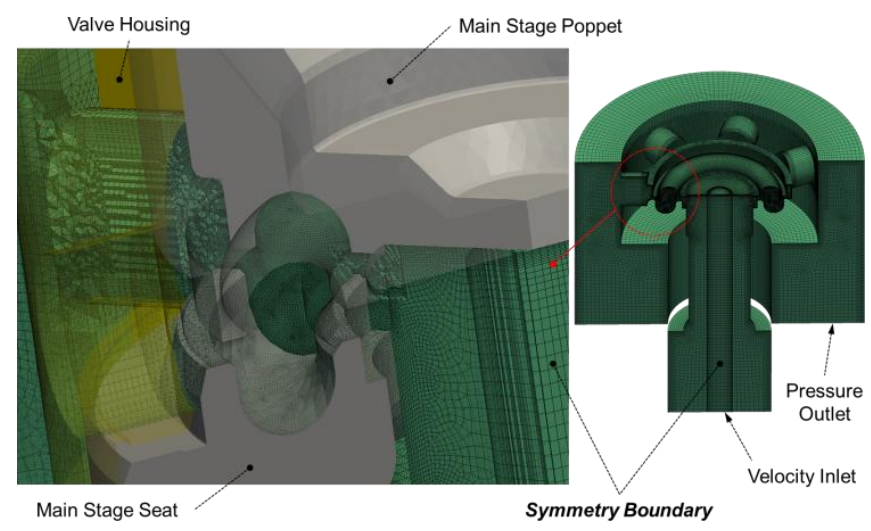

Figure 6: Main stage hybrid fluid mesh with symmetric boundary and zoomed clipped view on critical region

The accuracy and quality of the CFD analysis is validated against flow-bench experiments replicating exactly the CFD model setup. In particular, simulations with the poppet at fixed discrete positions have been compared with measurements, as shown by Figure 7. The pressure-flow characteristic of the main stage is represented by the total pressured drop between inlet and outlet boundaries. It should be noticed how the accuracy of the CFD modeling is strongly improved by the introduction of a multi-phase model, which models the gas entrainment in the fluid and its impact on the inlet pressure build-up. In particular, the Zwart-Gerber mixture model is used, with a saturation pressure of 1.0e4 $\mathrm{Pa}$. Gas entrainment effects are amplified by the unconventional main stage geometry: high speed vortical flows in the toroidal regions induce local static pressure drops, influencing the fluid phase volume fractions and the overall main poppet dynamics.

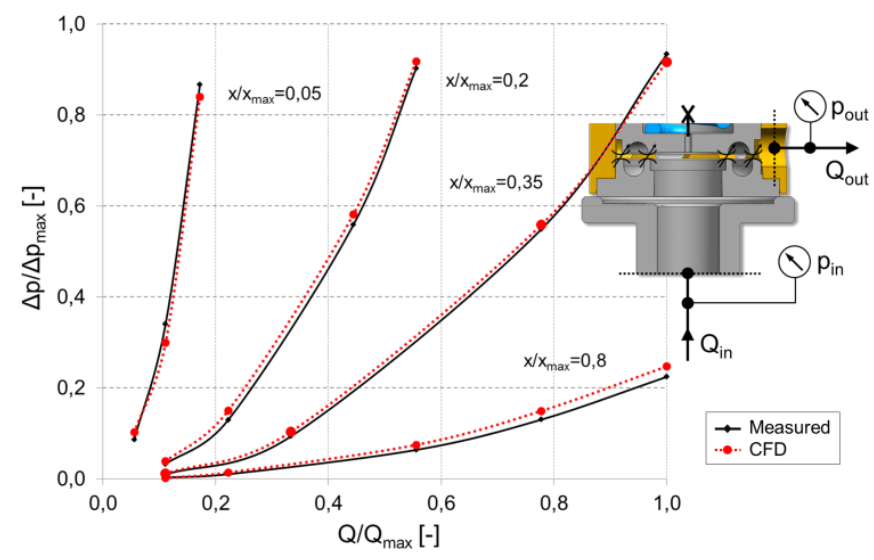

Figure 7: $C F D$ and measured total $p-Q$ characteristics across the main stage at different fixed positions

Typical CFD analysis results from a main stage simulation are shown by Figure 8 , where the complexity of the fluid field and its influence on the variable hydraulic volumes pressure build up can be noticed.
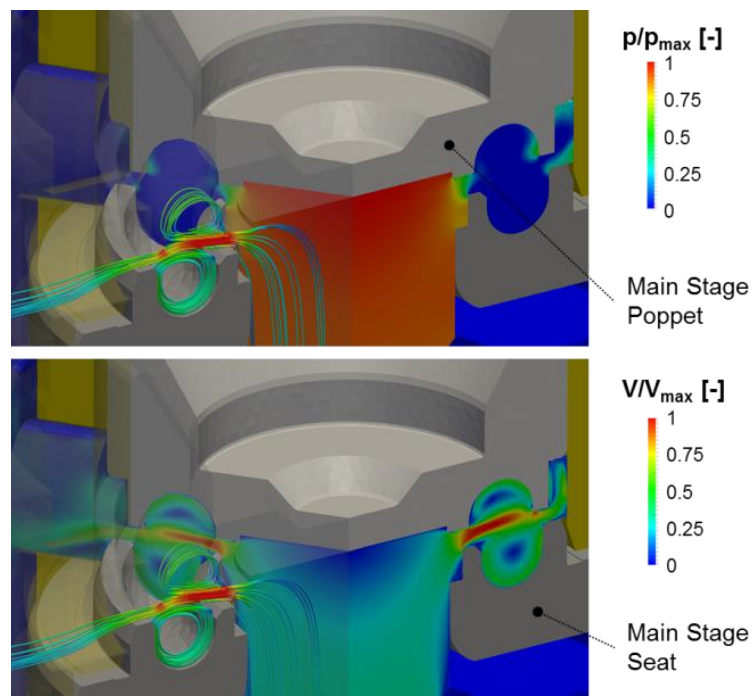

$\mathrm{V} / \mathrm{V}_{\max }[-]$

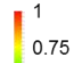

0.5

0.25

Main Stage Seat

Figure 8: Main stage CFD static pressure (top) and velocity (bottom) fields for one opening position

A combination of vortical flow fields, pressure stagnation regions and fluid jet interference on the moving element surfaces make CFD studies necessary to improve the lumped parameter model. In the main stage AMESim lumped parameter model, the flow rate calculations for each 
annular metering orifice are based on the Bernoulli turbulent equation, as shown by Equation 1 .

$$
Q=C_{\mathrm{q}}(x) \Omega(x) \sqrt{\frac{2|\Delta p|}{\rho}} \operatorname{sign}(\Delta p)
$$

Where the flow coefficient $C_{\mathrm{q}}$ is expressed as an hyperbolic tangent dependent on the main poppet lift, $x$, as follows:

$$
C_{\mathrm{q}}(x)=C_{\mathrm{q}}^{\infty}(x) \tanh \left(\frac{2 x}{x_{\text {crit }}}\right)
$$

The infinite flow coefficient $C_{\mathrm{q}}^{\infty}$ corresponds to the Von Mises asymptote or fully turbulent flow, while the critical poppet lift, $x_{\text {crit }}$, represents a value at which separation between laminar and turbulent flow occurs [13]. The use of a hyperbolic tangent allows a smooth transition between the two separate regimes.
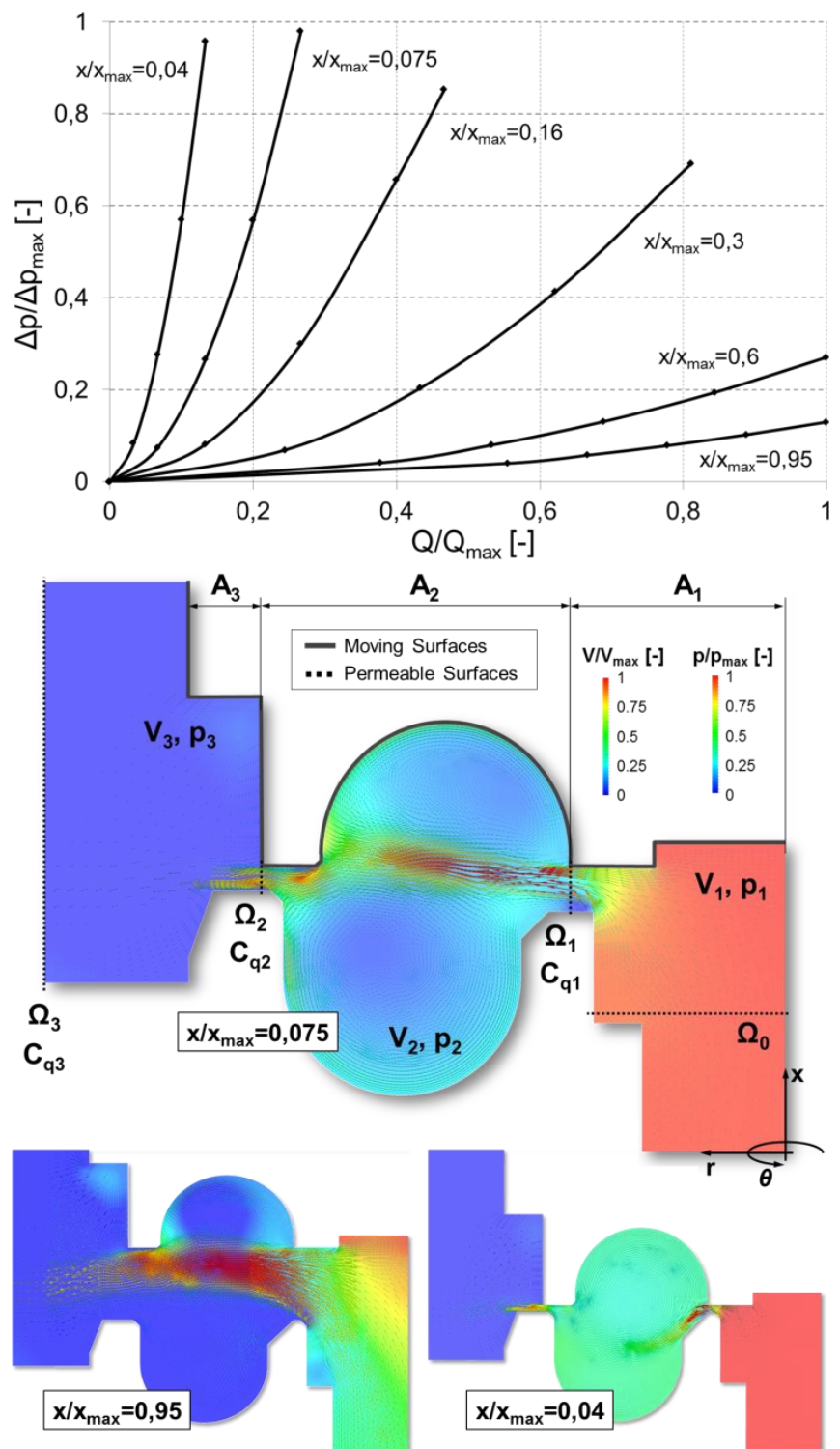

Figure 9: Main stage p- $Q$ curves from $C F D$ analysis (top), critical parameters on $1 D$ control volumes (center) and CFD flow fields at different openings (bottom)
Using the pressure-flow CFD simulation results as a reference and studying the flow field properties, shown by Figure 9, the values of the flow coefficients on the inner and outer restrictions are extracted for different poppet positions. Being the pressure-flow characteristics turbulent at all openings, the critical poppet lift number is kept constant at 1.0e-6. Referring to Figure 9, the AMESim model considers the pressure build-up in the separate control volumes characterizing the main stage. Hence, the inner and outer annular restrictions are treated separately. The main stage flow coefficients are expressed as a function of the main poppet lift, as depicted by Figure 10.

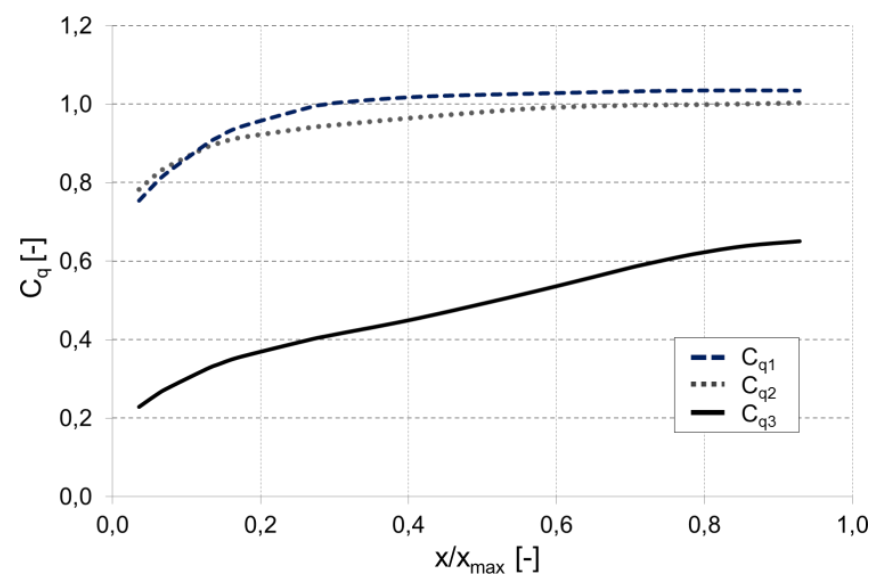

Figure 10: Main stage flow coefficients as a function of the main poppet lift

The flow coefficients curves are implemented in the 1D AMESim model to fit the pressure-flow characteristics predicted by the CFD analysis. The enhancement of the main stage lumped parameter 1D model in the pressure-flow calculations after the introduction of the CFD derived flow coefficients is shown by Figure 11.

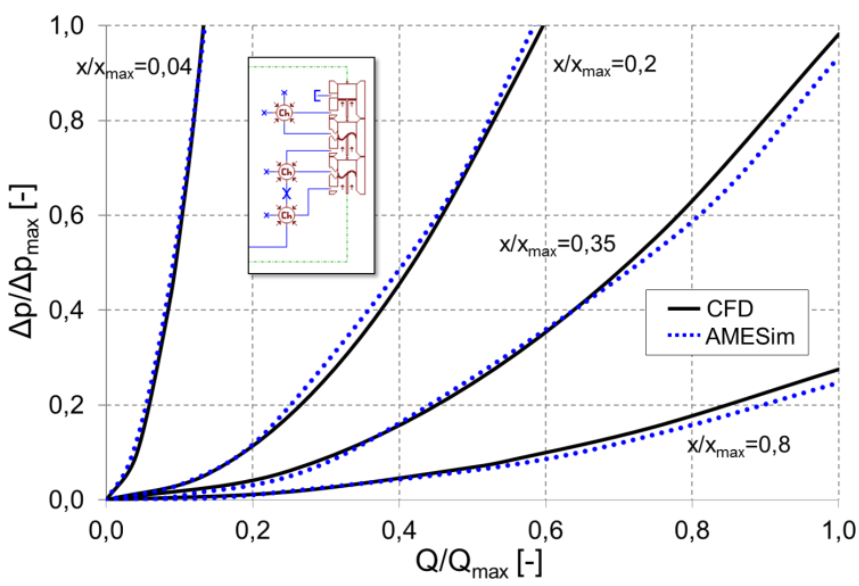

Figure 11: Main stage AMESim model pressure-flow characteristics compared with CFD results

Another critical aspect related to the lumped parameter modeling of hydraulic components is the influence of fluid inertia on the static pressure distributions, which ultimately govern the moving elements second order dynamics. The pressure stagnation phenomena, the non-homogenous 3D flow fields and the real separation between the control volumes pressure distributions, significantly affect the moving element active surfaces. For clarity, he method 
implemented for the determination of the hydrodynamic forces acting on the moving elements, based on the discussed CFD analysis, is outlined in the next section concerning the pilot stage modeling.

\subsection{Pilot Stage}

The pilot stage fluid domain is simplified taking advantage of the cyclical geometric design. In particular, a threedimensional arc of $120^{\circ}$ is considered. A single phase modeling of the fluid is considered, hence no air release and/or cavitation models are used. A pictorial representation of the fluid domain within the pilot stage geometry is given by Figure 12. It should be noticed how the pressure regulation is performed by a special shim spring, which simultaneously performs elastic and metering functions.

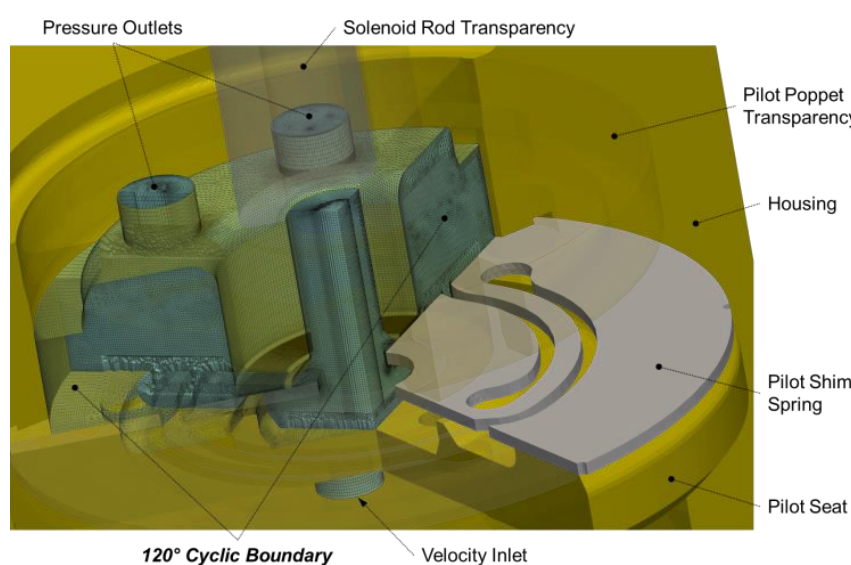

Figure 12: Pilot stage fluid hybrid mesh with cyclic periodicity clipped view for one opening position

Typical results from the CFD study at a given pilot poppet/shim spring position are shown in Figure 13, where the static pressure distribution on the pressurized shim surface, the pressure build-up upstream the annular metering region and the velocity field have to be considered in the coupling between $3 \mathrm{D}$ and $1 \mathrm{D}$ models.

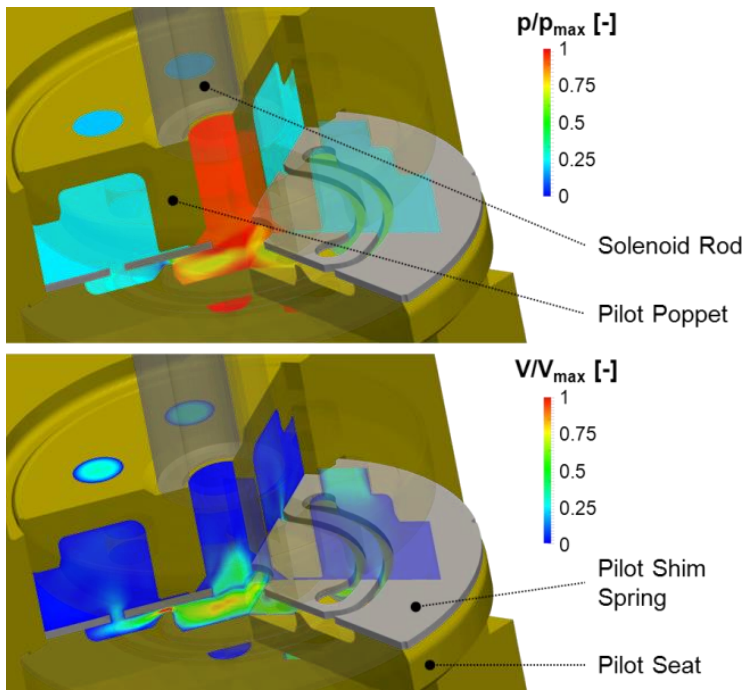

Figure 13: Pilot stage CFD static pressure (top) and velocity (bottom) fields for one opening position
Several operating points are considered in terms of poppet/shim positions, i.e. metering openings, and inlet flow rate, to extract the most critical fluid flow variables to be implemented in the 1D model. Similarly to the main stage analysis, pressure-flow characteristics for the pilot stage can be extracted from the CFD simulations, as depicted by Figure 14.

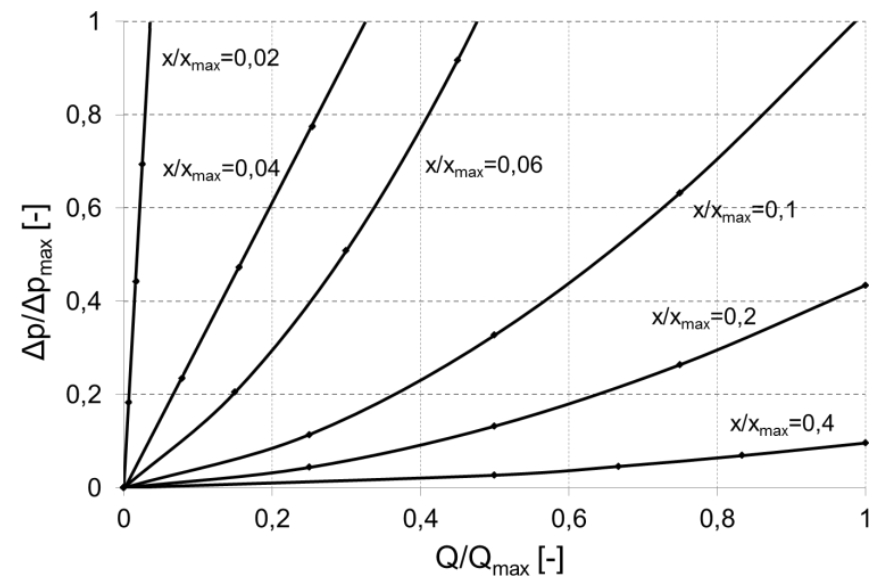

Figure 14: Pilot stage p-Q curves from CFD analysis in the small stroke range

Referring to Figure 14, due to the long overlap between pilot shim and pilot seat generating a thin annular gap, the transition between laminar to fully turbulent flow characterizes at least the first part of the stroke. Laminar CFD simulation in this range have been perforemd to confirm this behavior. This working area is critical for the pilot stage and overall valve dynamics, hence a correct modeling of the transition is a key element to predict the CES8700 physical behavior. For this reasons, in the pilot stage 1D model the Bernoulli orifice equation, Equation 1, is modified to better approximate the $p$ - $Q$ characteristics predicted by the CFD analysis. In particular, the following relationship is implemented:

$$
Q=K_{\mathrm{q}}(x) \Omega(x) \sqrt[n]{\frac{2|\Delta p|}{\rho}} \operatorname{sign}(\Delta p)
$$

Where $K_{\mathrm{q}}$ and $n$ are a special flow coefficient and a flow exponent respectively.

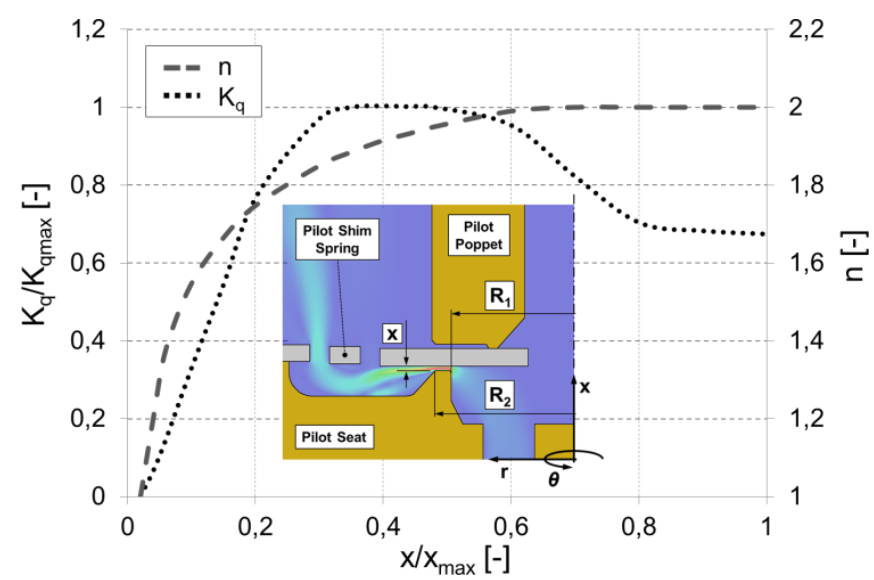

Figure 15: Pilot stage flow coefficient and flow exponent function of poppet/shim position 
Their behavior over the pilot poppet/shim stroke is derived from the CFD simulations results and is represented in Figure 15.

Referring to Figure 15, it can be noticed how in the laminar region the flow exponent reaches the value of 1 , while the flow coeffcient strongly reduces its value, approximating Equation 3 to the typical laminar Poisueille flow equation for an annular metering gap, shown by Equation 4 .

$$
Q_{\text {lam }}=\frac{\pi}{6 \mu \ln \left(\mathrm{R}_{2} / \mathrm{R}_{1}\right)} x^{3} \Delta p
$$

The flow parameters derived from CFD are fed as input to a special AMESim in-house developed model, which replicates the pilot stage flow behavior predicted by the CFD analysis, as shown by Figure 16.

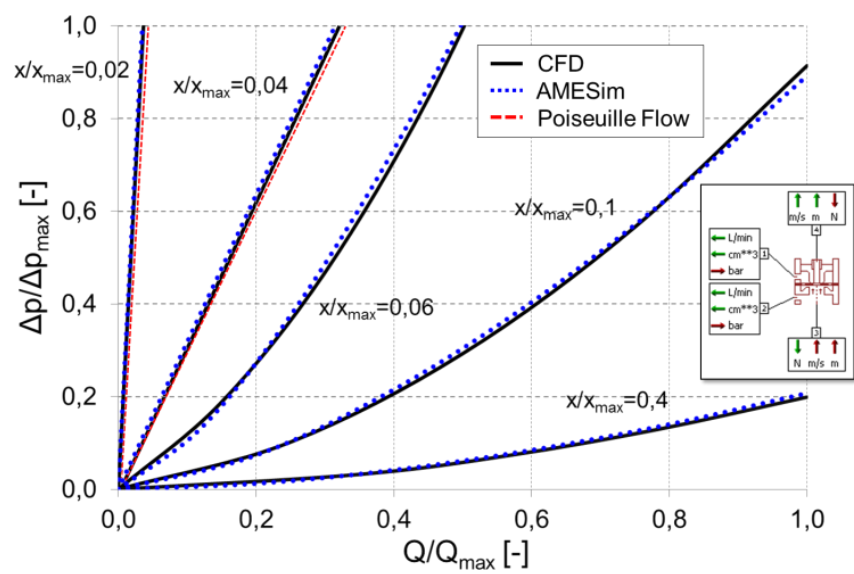

Figure 16: Pilot stage 1D-3D AMESim model pressure-flow characteristics compared with CFD results

As already mentioned in the previous section on the main stage, a critical aspect of lumped parameter modeling of hydraulic valves is represented by the prediction of the hydrodynamic forces acting on the moving elements. In fact, the pressure in each control volume is not uniform, since the fluid momentum causes local variations of static pressure distribution. Hence, the real hydraulic pressure forces acting on the moving bodies can be derived with the contribution of CFD analysis. In the pilot stage, the significant overlap characterizing the shim spring/pilot seat annular orifice together with the 3D fluid jet interactions with the shim body make the static pressure fields and the consequent opening pressure forces a critical physical parameter to be determined. Referring to Figure 17 and focusing on the annular metering orifice region, where the flow field approximates axisymmetric conditions, part of the hydrodynamic forces evaluation is can be based on the classic flow force expression, deriving from the integration of the momentum equation over the system control volume. Assuming incompressible, steady-state conditions, the general expression for the active hydraulic force acting on the poppet/shim assembly leads to:

$$
\left.\vec{F}_{\text {hyd }}\right|_{x}=p_{0} A_{0}+p_{1} A_{1}-\rho Q\left(\vec{U}_{1} \cos \delta_{1}-\vec{U}_{0} \cos \delta_{0}\right)
$$

Where $\vec{U}$ is the fluid velocity vector and $\delta$ is the fluid jet angle formed with the $x$-axis. Thanks to the overlapped design on the restriction, the jet angle is not varying significantly over the stroke, being $\delta_{1}$ consistently close to $90^{\circ}$, especially at the smaller openings. However, the impact of fluid momentum on the effective hydraulic forces is known to be significant, hence the equivalent representation of the separation between the two static pressure fields upstream and downstream the pilot restriction has to be improved [14].

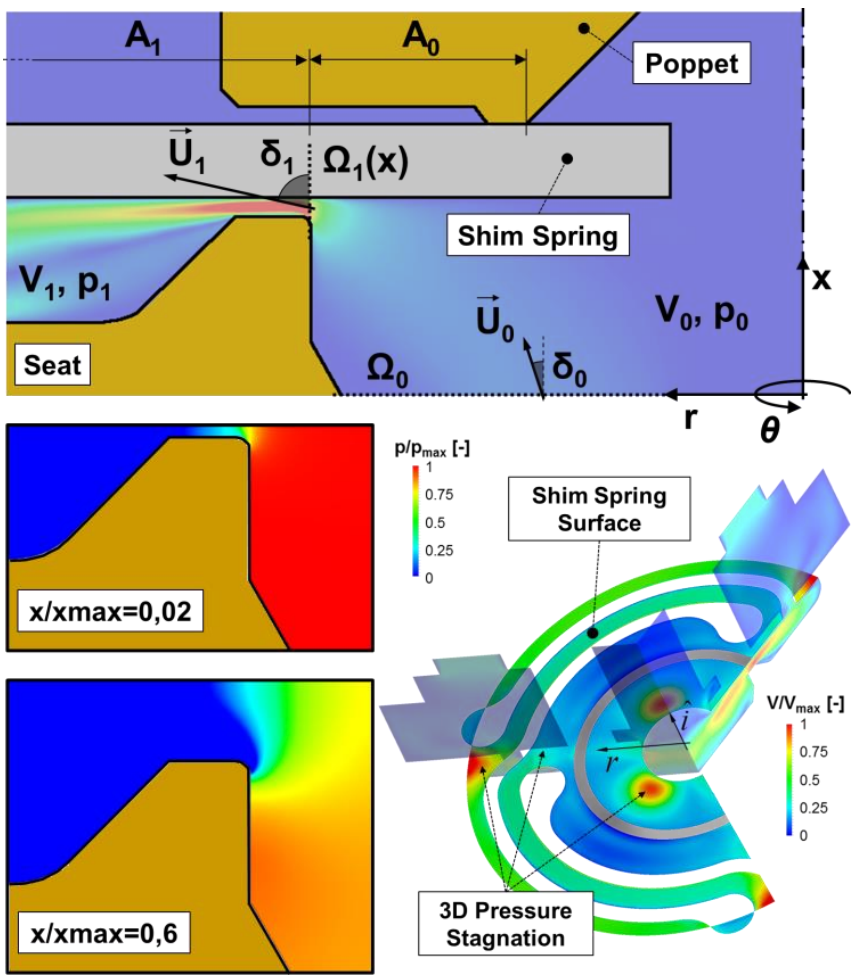

Figure 17: Pilot stage critical parameters on $1 D$ control volumes (top) and different critical CFD flow field characteristics (bottom)

The active pressurized areas are not the nominal ones and as shown by Figure 17, 3D pressure stagnation effects varying over the poppet stroke influence the poppet/shim dynamics. The equivalent upstream and downstream active areas are derived from the CFD analysis performed on the pilot stage. In particular, simplifications on Equation 5 are made, by considering gauge pressure $p_{1}$ as $0[\mathrm{~Pa}]$, by neglecting the inlet velocity $\vec{U}_{0}$ and by focusing on the inertia driven turbulent flow, since at the smallest openings $\delta_{1} \approx 90^{\circ}$. Based on these assumptions, it is possible to derive an equivalent shim pressurized area as follows:

$$
A^{*}=\frac{\int_{A_{\mathrm{shim}}} p_{\operatorname{shim} \mathrm{CFD}} \cdot \hat{i} d A+2 K_{\mathrm{q}}(x) \Omega(x) \cos \delta_{1}}{p_{0}}
$$

Thanks to the CFD simulation campaign performed it is possible to study the variation of $A^{*}$ as a function of the poppet stroke and the pilot stage pressure differential, as shown by Figure 18. Referring to Figure 18, the unique shape of the shim active pressurized area does not depend strongly on the pressure difference, but mostly on the stroke. Hence, to remove one degree of freedom in the 1D model, it 
is assumed that the static pressure distribution on the shim spring is purely a function of the geometrical characteristic of the restriction and an average $A^{*}$ curve is implemented. Referring to Figure 17, at the smallest openings the upstream pressure enters the annular orifice, maximizing the pressurized area. However, as the increment of the stroke opens the annular restriction, the pressure drop takes place further away from it, reducing the effective pressurized region. At the higher stroke values, the 3D pressure stagnation phenomena contribute to an increment of the equivalent active area.

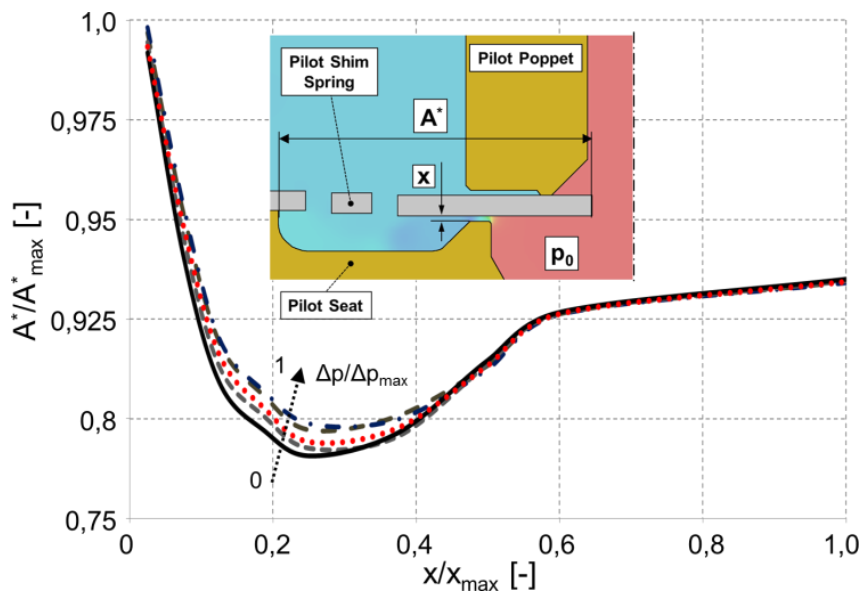

Figure 18: Effective shim spring pressurized area function of pilot poppet position at different pressure drops

The effective shim active area curve is an input to the pilot stage AMESim model, so that the coupling of 3D extrapolated data with the 1D model allows a precise prediction of the hydraulic pressure forces acting on the pressurized surfaces, as shown by Figure 19.

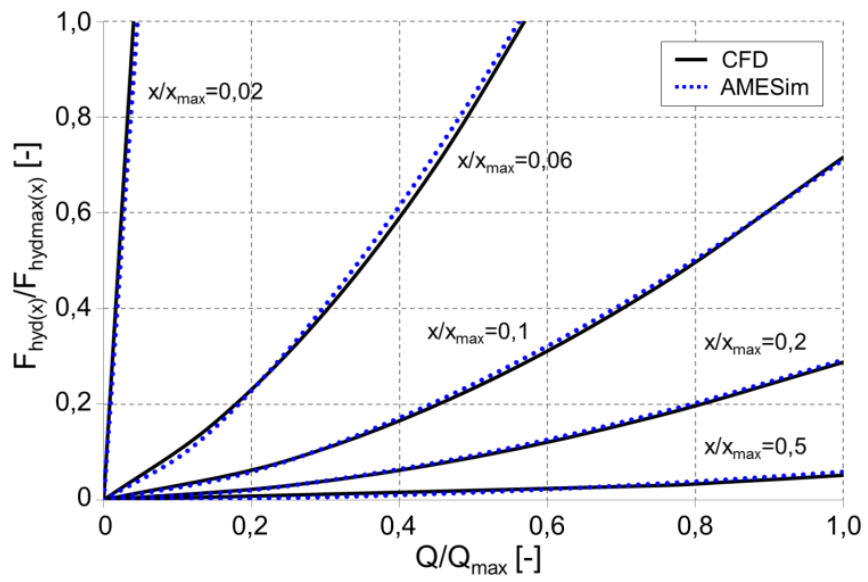

Figure 19: Pilot stage custom 1D-3D coupled AMESim model hydraulic forces compared with CFD results

\section{The 1D AMESim Model}

Lumped parameter modeling with AMESim allows capturing the dynamics of the CES8700 physical system. The main external forces acting on the inertial equilibrium of the pilot stage and main stage moving elements are considered, i.e. friction forces, pressure forces, spring forces, solenoid forces, etc. An overview of the CES8700 AMESim model is given in Figure 20.

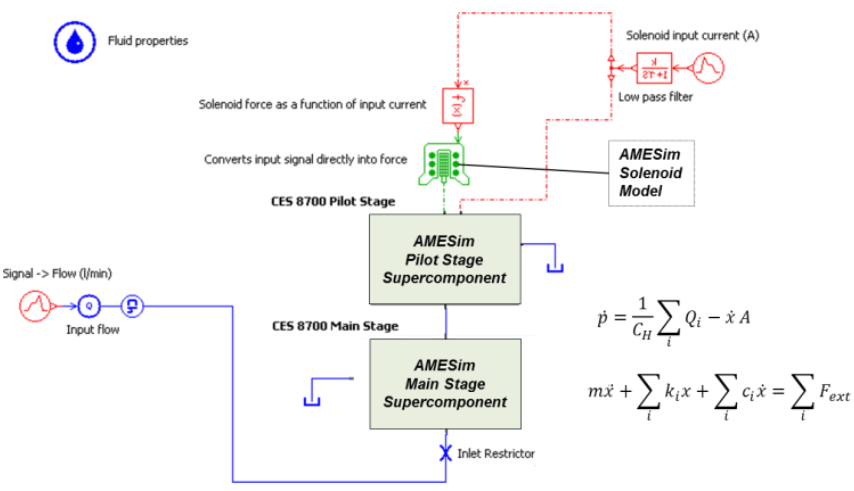

Figure 20: The CES8700 LMS AMESim model

AMESim super-components are implemented; lumping customized components to include the numerical results from 3D modeling. In particular, the customized components allow solving for the variable volumes pressure build-up equation, while considering the flow coefficients derived from CFD analysis in the turbulent flow orifice equations. Furthermore, a critical variable for the correct equilibrium of the moving elements is represented by the flow force. The flow forces acting on the pressurized surfaces are considered by implementing the CFD results as a function of the moving elements position. The 1D model replicates the experimental flow-bench setup of the CES8700 valve during testing, where the inputs to the system is a saw tooth inlet flow rate signal, corresponding to progressively increasing levels of solenoid current.

\section{Coupled Numerical Results}

The aim of this paper is to show how, by coupling 3D numerical data with a 1D lumped parameter model, a more accurate prediction of the whole dynamic behavior of the CES8700 hydraulic valve can be achieved. In particular, by extracting turbulent flow coefficients and information about static pressure distributions on the moving elements, further understanding on the valve dynamics is reached.

Numerical results are compared with measurements, in order to assess the accuracy of the developed 1D-3D coupled model. In particular, inputs to the 1D lumped parameter model are a flow rate signal and a solenoid current signal, as shown by Figure 21 .

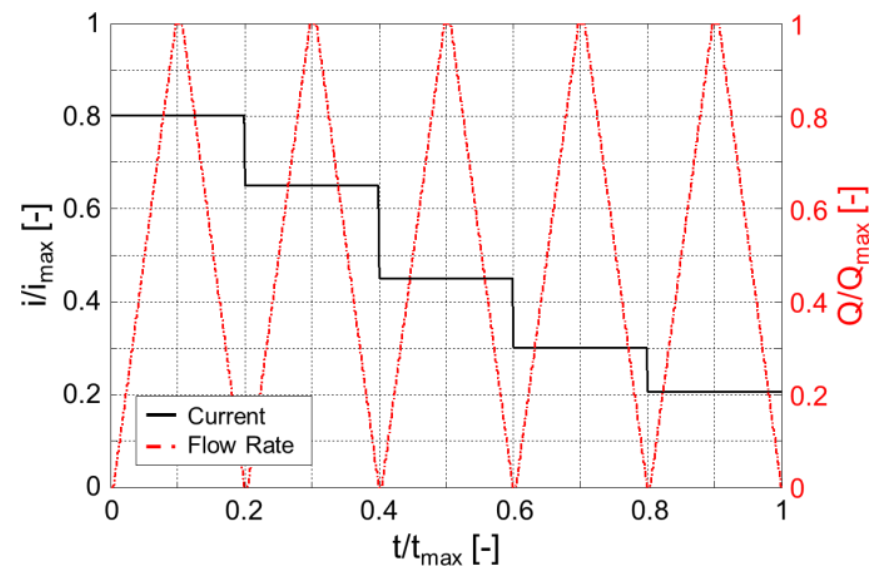

Figure 21: Input signals to $1 D$ simulation models 
Additionally, the flow benches available at the Öhlins CES Technologies facilities allow testing the pilot stage alone as well as the full assembled valve, composed by main and pilot stages. Hence, in the next paragraphs an overview of the numerical results compared with measurements is given, both for the pilot stage and the full CES8700 valve.

\subsection{Pilot Stage}

Referring to Figure 22, comparison between measurements on the CES8700 pilot stage and two different 1D model combinations is shown. In particular, the 1D-3D coupled model presented in this paper and a similar AMESim model based on standard components, implementing optimized constant flow coefficients and constant flow force jet angles, are considered. It can be noticed how the numerical results of the 1D model enhanced by CFD data are more accurate over the entire range of flow rates and input currents. The remaining discrepancies between measured and simulated data are affected by measurements scatter and complex friction hysteresis phenomena, not yet precisely considered in the $1 \mathrm{D}$ model.

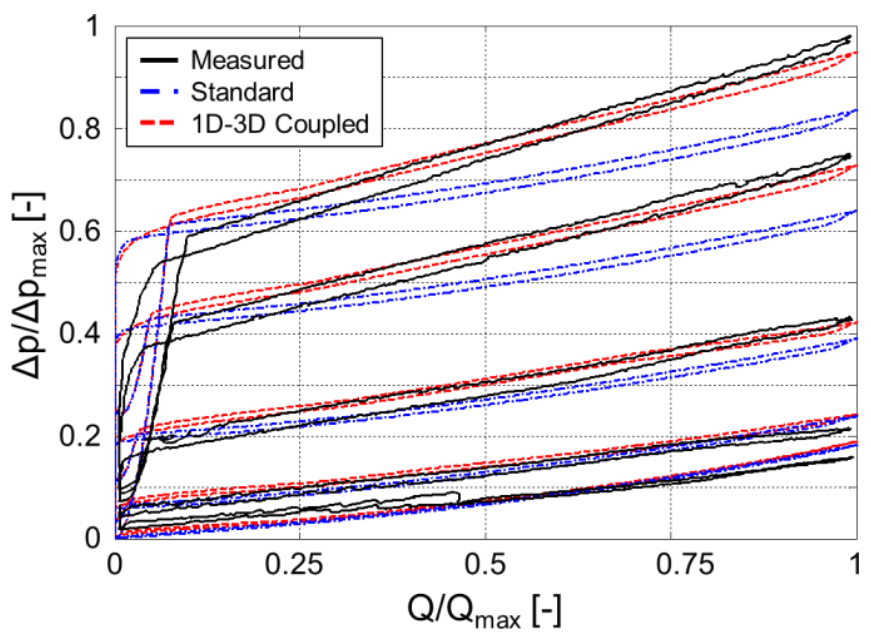

Figure 22: Pilot stage pressure levels from the two $1 D$ models compared with flow bench measurements

Referring to Figure 22, each pressure level corresponds to a specific solenoid current step, in particular the higher the current the higher the pressure drop across the pilot stage.

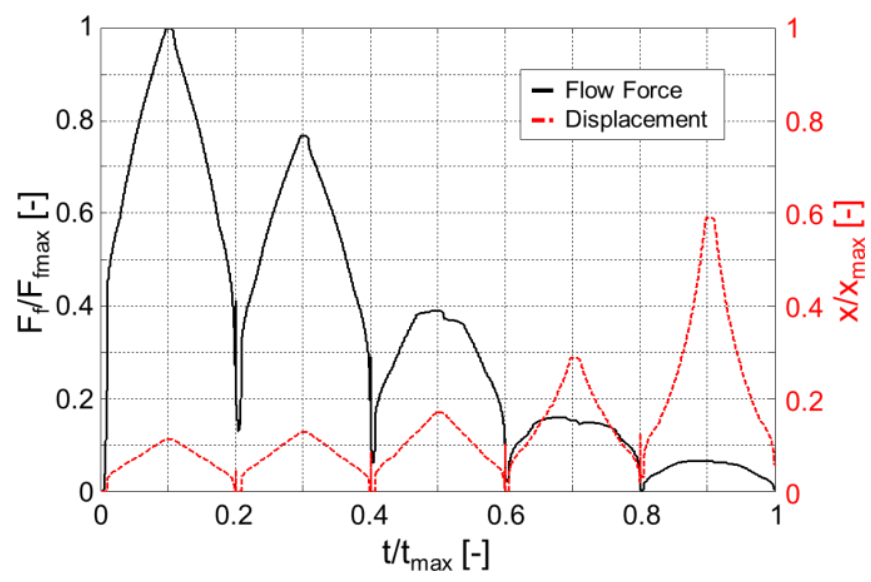

Figure 23: Pilot stage predicted flow forces and pilot poppet displacement for $1 D-3 D$ coupled model
Insights on the pilot stage dynamics can be extracted from the lumped parameter modeling. For example, accurate predictions on the total flow forces magnitude, i.e. difference between nominal and effective pressure forces, as well as the corresponding pilot poppet displacement are calculated by the 1D-3D coupled model, as shown by Figure 23. Referring to Figure 23, the pilot poppet/shim assembly displacement increases as the solenoid current progressively decreases, allowing a higher efflux area for the flow rate and consequently a lower pressure level upstream the pilot stage. The gradually lower pressure drop across the pilot metering orifice induces a lower flow force.

\subsection{Full Valve}

Similarly to the pilot stage, the lumped parameter model of the complete CES8700 valve is compared with measurements. Thanks to the flow-bench measurements comparisons, the accuracy of the developed simulation model and of the numerical strategies presented in this paper is validated.

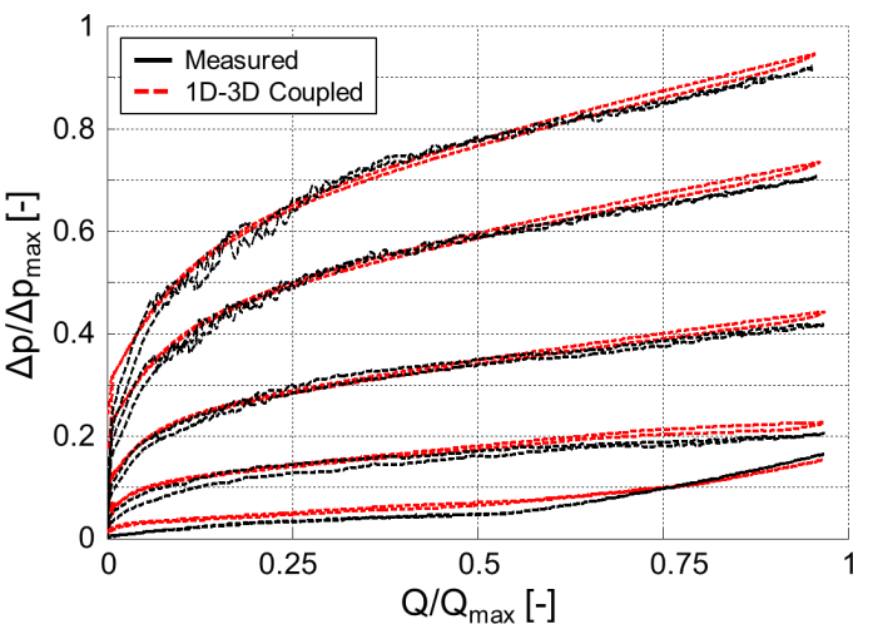

Figure 24: CES8700 p-Q characteristics from 1D-3D model and flow-bench measurements

Referring to Figure 24, the comparison between measured CES8700 $p$ - $Q$ characteristics at different current levels and the predicted valve behavior from the $1 \mathrm{D}-3 \mathrm{D}$ coupled model is shown.

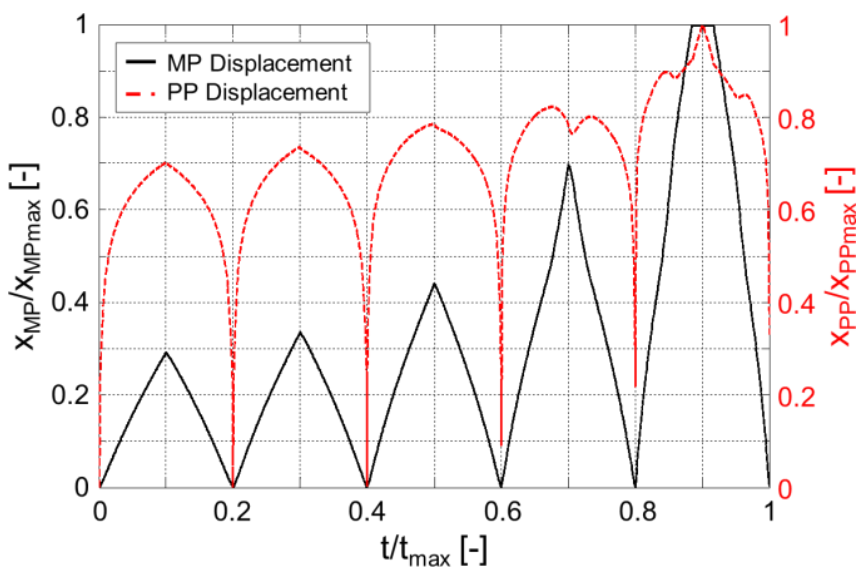

Figure 25: Main poppet and pilot poppet displacement from ID-3D coupled model 
Following the pilot stage behavior, higher pressure levels correspond to higher input solenoid currents. The lumped parameter model of the solenoid valve allows calculating physically inaccessible parameters, impossible to measure, such as the main and pilot poppets simultaneous displacements, depicted by Figure 25. It can be noticed how the poppets

\section{Conclusions}

A numerical analysis on a special solenoid hydraulic valve for semi-active suspensions is presented in this paper. The valve, designed by Öhlins CES Technologies, is in use by several major automotive manufacturers to implement semiactive shock absorbers technology in their vehicles. The unconventional design of the valve, named CES8700, requires advanced computer simulation to predict its hydraulic dynamic behavior. An extensive CFD analysis on the most critical regions of the CES valve is discussed, providing experimental validation on specific regions of the hydraulic valve. The distributed parameter modeling is not only necessary to understand the impact of key design features, but also to extract physical variables associated with the fluid flow. In particular, flow coefficients and static pressure distributions on the moving elements are studied. These parameters are coupled with a 1D numerical model of the valve, used to predict the whole system dynamic behavior. The influence of the CFD results on the 1D model numerical results is discussed. It is shown, comparing simulation results with experimental measurements, how the information from a detailed CFD analysis strongly improves the ability to fully capture the CES hydraulic valve behavior. In the future, 2-way and flow-driven rigid body FSI (Fluid-Structure Interaction) analysis will be developed; in order to understand the impact of the elastic components interaction with the fluid flow and to capture the effects of localized fluid flow phenomena on the poppet dynamics.

\section{References}

[1] Blackburn, J. F., Reethof G., Shearer, J. L. 1966. Fluid Power Control, USA: MIT Press.

[2] H E Merritt. 1967. Hydraulic Control Systems. John Wileys and Sons, Cincinnati, Ohio, ISBN 0-471-596175.

[3] Nervegna, N. 2003. Oleodinamica e Pneumatica: Sistemi, Componenti, Esercitazioni. Politeko, Turin, Italy.

[4] Borghi, M., Cantore, G., Milani, M. and Paoluzzi, R. 1998. Analysis of hydraulic components using computational fluid dynamics models. Proceedings of the Institution of Mechanical Engineers, Vol. 212 (7) Part C, pp. 619-629.

[5] Vacca, A., Greco M., Fornaciari, A. 2008. New concept for the development of a relief, anti-shock and anticavitation cartridge valve. Proceedings of the 2008 IFK, Dresden, Germany.
[6] Amirante R, Del Vescovo G, Lippolis A. 2006. Evaluation of the flow forces on an open centre directional control valve by means of a computational fluid dynamic analysis. Energy Conversion \& Management 47, pp. 1748-1760.

[7] Amirante R, Del Vescovo G, Lippolis A. 2006. A flow forces analysis of an open center hydraulic directional control valve sliding spool. Energy Conversion \& Management 47, pp. 114-131.

[8] Amirante, R., Moscatelli, P. G., and Catalano, L. A. 2007. Evaluation of the Flow Forces on a Direct (Single Stage) Proportional Valve by Means of a Computational Fluid Dynamic Analysis. Energy Conversion \& Management 48, pp. 942-953.

[9] Del Vescovo G, Lippolis A. 2003. CFD analysis of flow forces on spool valves. Proceedings of the $1^{\text {st }}$ International Conference on Computational Methods in Fluid Power Technology, Melburne, Australia.

[10] Valdés, J. R., Miana, M. J., Núñez, J. L., and Pütz, T. 2008. Reduced Order Model for Estimation of Fluid Flow and Flow Forces in Hydraulic Proportional Valves. Energy Conversion \& Management 49, pp. 1517-1529.

[11] Ansys Fluent User's Guide, Release 14.0, November 2011.

[12] Ansys Fluent Theory Guide, Release 14.0, November 2011.

[13]LMS Imagine AMESim Rev. 11 Reference Manual 2012.

[14]Clavier, A., Alirand, M., Vernant, F. and Sagot, B. 2003. Local Approach to Improve the Global Approach of Hydraulic Forces in Ball Poppet Valves. Proc. of the $4^{\text {th }}$ International Symposium on Fluid Power, Wuhan, China.

[15] Baudry X, Mare JC. 2000. Linking CFD and lumped parameters analysis for the design of flow compensated spool valves. Proceedings of the $1^{\text {st }}$ FPNI-PhD symposium, Hamburg, Germany, pp. 249-258.

[16]Borghi M, Milani M, Paoluzzi R. 2005. Influence of notch shape and number on the metering characteristics of hydraulic spool valves. International Journal of Fluid Power, Vol.6 (2), pp. 5-18.

[17] Borghi M, Milani M, Paoluzzi R. 2000. Stationary axial flow force analysis on compensated spool valves. International Journal of Fluid Power, Vol. 1(1), pp. 17-25. 